\title{
Spinal ependymomas. Part 2: Ependymomas of the filum terminale
}

\author{
Jörg Klekamp, MD \\ Department of Neurosurgery, Christliches Krankenhaus, Quakenbrück, Germany
}

OBJECT Ependymomas of the filum terminale provide specific surgical challenges due to their often enormous size, contact with nerve roots of the cauda equina and conus, and potential for subarachnoid dissemination. This study presents treatment results for these tumors over a 30-year period.

METHODS Among 1447 patients with tumors of the spinal canal treated between 1980 and 2014, 618 patients presented with extramedullary tumors. Of these, 42 patients ( 25 males, 17 females) demonstrated a myxopapillary ependymoma in the lumbosacral region. Thirty-four patients underwent 36 operations for 39 such tumors. The mean patient age was $38 \pm 14$ years (range 11-73 years), with an average clinical history of $37 \pm 67$ months. Patients were followed through outpatient visits and questionnaires, with a mean follow-up of 10 years (127 \pm 100 months). Twenty-seven operations were performed to treat de novo tumors and the remainder were undertaken on recurrent tumors. Short-term results were determined for individual symptoms, and tumor recurrence rates were calculated with Kaplan-Meier statistical analyses.

RESULTS Subarachnoid dissemination was observed in 11 patients and was related to previous surgery in 9 patients and associated with extensive tumors in 2 patients. Gross-total resections (GTR) were achieved in 28 operations (77.7\%) and subtotal resections in the remainder. Subtotal resections were restricted to unencapsulated ependymomas (61.5\%). Radiotherapy was employed after 6 operations on unencapsulated tumors, with 5 of these also demonstrating subarachnoid seeding. Permanent surgical morbidity affected 3 patients who experienced permanent worsening of bladder function, while 7 patients showed no postoperative changes, and the remaining 26 operations were followed by improvements. Long-term outcome depended on the amount of resection and the presence of a tumor capsule. Eight of 9 tumor recurrences affected unencapsulated tumors, of which 3 had undergone GTR. The overall recurrence rates were $6.6 \%$, $19.0 \%$, and $37.0 \%$ after 1,10 , and 20 years, respectively. For unencapsulated ependymomas, the corresponding rates were $15.6 \%, 32.5 \%$, and $66.2 \%$ after 1,10 , and 20 years, respectively, with significantly lower rates of $9.1 \%$ after 10 and 20 years for encapsulated tumors. Postoperative radiotherapy tended to prolong the recurrence-free interval for patients with unencapsulated tumors. Five patient deaths occurred during follow-up, of which 2 deaths were tumor related and occurred at 216 and 287 months after surgery.

CONCLUSIONS Extramedullary ependymomas are slow-growing tumors in the lumbosacral region, sometimes with an indolent course for long periods of time. Despite their delicate location and often enormous size, surgical morbidity in experienced hands is low, with good chances for postoperative clinical improvements and very low recurrence rates after GTR for encapsulated tumors. The role of postoperative radiotherapy remains controversial. Radiotherapy may be considered after incomplete resections of unencapsulated tumors and/or for patients with subarachnoid dissemination.

http://thejns.org/doi/abs/10.3171/2015.5.FOCUS15151

KEY WORDS extramedullary tumors; myxopapillary ependymomas; spinal tumors

$\mathrm{E}$ XTRAMEDULLARY ependymomas are rare, slow-growing pathologies that originate from the filum terminale and may reach an enormous size, sometimes filling out the entire lumbosacral canal. They may cause atrophy of the dura and display extradural extension with local bone destruction. Their often indolent course, despite their considerable size, and the risks of damage to nerve roots or the conus of the spinal cord may lead patients to hesitate to opt for surgery.

A controversial aspect of treatment is the role of postoperative radiotherapy. Does it have an effect on these slow-growing tumors? Should we reserve it for patients

ABBREVIATION GTR = gross-total resection .

SUBMITTED March 30, 2015. ACCEPTED May 19, 2015.

INCLUDE WHEN CITING DOI: 10.3171/2015.5.FOCUS15151.

DISCLOSURE The author reports no conflict of interest concerning the materials or methods used in this study or the findings specified in this paper. 
after subtotal removals or for patients with subarachnoid tumor dissemination?

This paper analyzes the results for 42 patients with these tumors who underwent treatment during 3 decades, from 1982 to 2014.

\section{Methods}

A total of 1447 patients with tumors of the spinal canal presented at the Nordstadt Hospital in Hannover, Germany (from 1980 to 2003), or the Christliche Krankenhaus in Quakenbrück, Germany (from 2004 to 2014), and were entered into the spinal cord database. All patients agreed in writing upon admission that their medical data may be used for scientific purposes in anonymous form. The first patient with an ependymoma of the filum terminale presented in 1982, marking the beginning of the study period. Data prior to 1991 were collected retrospectively (5 patients with this pathology). As of 1991, all patients were entered in the database continuously. Apart from general patient data and specific features of each spinal cord pathology, the results of neurological examinations before surgery, before discharge from the hospital, after 3 months postoperatively, and yearly thereafter were analyzed for individual symptoms according to a scoring system. ${ }^{20-22}$ Follow-up information was obtained through outpatient visits or questionnaires. Among these 1447 patients, 618 patients presented with extramedullary tumors. Of these, 42 patients were found to have an ependymoma of the filum terminale, representing $2.9 \%$ of all spinal, $4.5 \%$ of all intradural, and $6.8 \%$ of all extramedullary tumors diagnosed during this period. There were 25 males and 17 females. Unless a patient appeared unfit to undergo an operation, surgery was recommended to all symptomatic patients presenting with this tumor de novo. In patients with recurrent tumors, surgery was recommended to patients with evidence of tumor growth or progressive neurological symptoms. All 42 patients fulfilled criteria to be recommended for surgery. Eight patients, however, declined surgery while 34 patients underwent 37 operations for a total of 39 tumors.

In 1 patient who presented before the introduction of MRI, the diagnosis was based on CT with contrast and myelography, and in the remaining patients the diagnosis was based on MRI findings.

All operations were undertaken with the patient in a prone position and with monitoring of somatosensory evoked potentials (Fig. 1). The exposure involved laminotomies, with reinsertion of the lamina performed for children and laminectomies employed for adults up to the mid-1990s. Thereafter, lamina were reinserted with titanium miniplates. The exact extension of the tumor was then demonstrated by ultrasonography before dura opening. The dura was opened in the midline and held open with sutures. It was the general policy of the 2 institutions involved in the series to remove these tumors in toto unless their size or adherence to nerve roots or the conus required debulking of the tumor first to preserve these structures. To prevent any subarachnoid spreading of tumor particles during debulking, small cotton patties were positioned around the entire tumor before starting the resection. This procedure also helped to prevent nerve inju- ries during further dissection. Once the tumor mass had been removed inside the capsule, the filum terminale was identified, coagulated, and cut. Lifting the proximal end of the filum together with the remaining tumor allowed the nerve roots on the ventral side to be mobilized, and additional cottonoids were placed to keep them separated from the remaining lesion. The cranial pole of the tumor was then dissected free to follow the ventral tumor side caudally to identify the exact position of the conus. Finally, the filum was transected immediately below the conus to achieve a complete tumor resection, i.e., gross-total resection (GTR). The dura was closed with a tight running suture. In patients undergoing surgery on a recurrent tumor, a duraplasty was inserted to minimize postoperative adhesions between conus and dura. In patients with atrophy of the inner dural layer, a lumbar drain was placed for CSF drainage for about a week to prevent CSF fistulas. The wound was closed in standard fashion. Starting in the early 1990s, predischarge MRI studies were routinely ordered. Radiotherapy was advised only for patients with incomplete tumor resections of unencapsulated tumors and/ or subarachnoid dissemination.

For statistical tests of significance, Student t-tests and chi-square tests were employed. Means are given with the standard deviation. Surgical morbidity was defined as a permanent aggravation or a new, permanent deficit within 30 days after the operation. Long-term follow-up results were analyzed with Kaplan-Meier statistics ${ }^{17}$ to determine the percentages of patients with tumor recurrences. Differences were considered statistically significant if a $p$ value $\leq 0.05$ was reached. Results of a multiple regression analysis are provided with $\beta$ values to indicate the impact of individual factors, the correlation coefficient of the regression equation, and its $\mathrm{p}$ value. The software package PC-Statistik, version 5.0, (Hoffmann-Software) was employed for all statistical analyses.

\section{Results}

Forty-two patients had a myxopapillary ependymoma in the lumbosacral region (Table 1). The mean patient age was $38 \pm 14$ years (range 11-73 years), with an average clinical history of $37 \pm 67$ months and a mean follow-up of $127 \pm 100$ months. In 34 patients tumors were restricted to a maximum of 3 vertebral levels (small tumor group) (Fig. 1), while in 8 patients ependymomas were spread over at least 4 segments (large tumor group) (Fig. 2).

Surgery was recommended to every patient of this series. Eight patients, however, declined to undergo surgery. Their average clinical history was considerably longer than that of patients accepting surgery $(112 \pm 126$ months vs $24 \pm 42$ months, not significant), which may explain their hesitation. Table 2 lists the percentages for individual neurological symptoms to provide an overview of the clinical spectrum. Pain was the predominating symptom for most patients.

For this investigation, tumors displaying a capsule at surgery $(\mathrm{n}=23)$ (Fig. 1) were distinguished from those without such a capsule $(n=16)$ (Fig. 2). Comparing these groups revealed more severe neurological deficits associated with the latter, and the difference reached a significant level for gait ataxia and sphincter disturbances (Table 

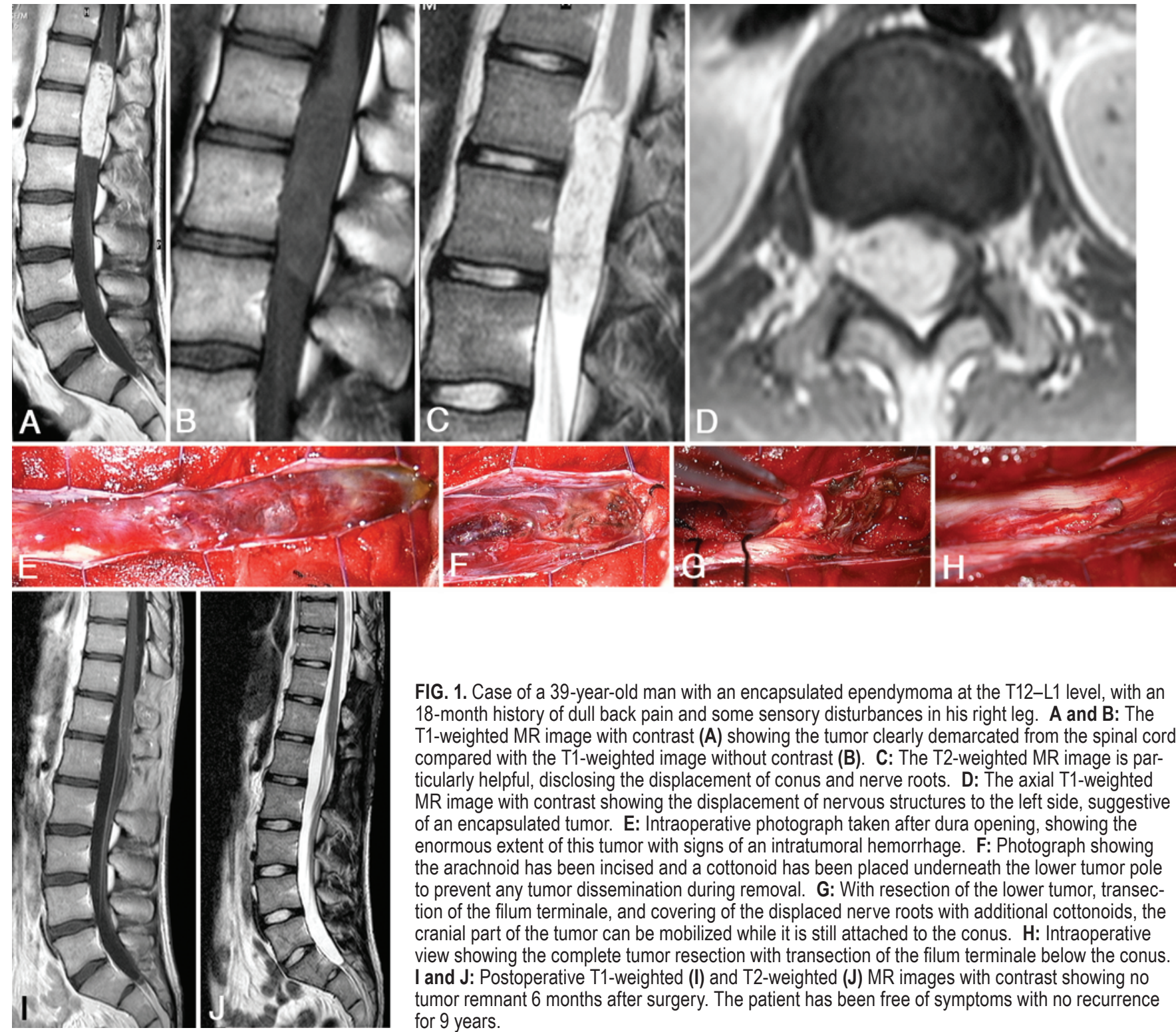

FIG. 1. Case of a 39-year-old man with an encapsulated ependymoma at the T12-L1 level, with an 18-month history of dull back pain and some sensory disturbances in his right leg. A and B: The T1-weighted MR image with contrast (A) showing the tumor clearly demarcated from the spinal cord compared with the T1-weighted image without contrast (B). C: The T2-weighted MR image is particularly helpful, disclosing the displacement of conus and nerve roots. D: The axial T1-weighted MR image with contrast showing the displacement of nervous structures to the left side, suggestive of an encapsulated tumor. E: Intraoperative photograph taken after dura opening, showing the enormous extent of this tumor with signs of an intratumoral hemorrhage. F: Photograph showing the arachnoid has been incised and a cottonoid has been placed underneath the lower tumor pole to prevent any tumor dissemination during removal. G: With resection of the lower tumor, transection of the filum terminale, and covering of the displaced nerve roots with additional cottonoids, the cranial part of the tumor can be mobilized while it is still attached to the conus. $\mathrm{H}$ : Intraoperative view showing the complete tumor resection with transection of the filum terminale below the conus. I and J: Postoperative T1-weighted (I) and T2-weighted (J) MR images with contrast showing no tumor remnant 6 months after surgery. The patient has been free of symptoms with no recurrence for 9 years.

2). Furthermore, the history of unencapsulated tumors tended to be slightly longer $(27 \pm 58$ months vs $22 \pm 29$ months; not significant) and their sizes were significantly larger $(p=0.05)$. All encapsulated tumors were limited to a maximum of 3 spinal levels, whereas half of the unencapsulated tumors extended over a minimum of 4 levels.

Subarachnoid dissemination was evident in 11 patients and was restricted to tumors without capsules and correlated with tumor size; among small tumors, no seeding was observed unless related to a previous incomplete resection, as seen in 6 instances. In the group of patients with large tumors, 6 of 8 patients presented with evidence of dissemination, 4 of them after a previous operation (Table 1).

Thirty-four patients underwent 36 operations for $39 \mathrm{ep-}$ endymomas. Twenty-seven operations dealt with de novo tumors and 9 operations were undertaken on recurrent lesions. A GTR was achieved in 28 operations (77.7\%), with subtotal resections in the remainder. All 23 encapsulated ependymomas were completely removed and required no adjuvant therapy (Fig. 1). Incomplete resections were restricted to unencapsulated tumors (61.5\%). Likewise, all but 1 of the tumors of small sizes were removed completely, irrespective of previous surgeries or presence of a tumor capsule, whereas 7 of 8 tumors spreading over a minimum of 4 spinal segments were removed subtotally due to arachnoid scarring from previous operations, subarachnoid dissemination, and/or adherence to important nerve roots (Fig. 2). In this series, 1 postoperative dissemination occurred among patients operated on for de novo tumors. Radiotherapy was employed after 6 operations, of which 5 had been undertaken on recurrent tumors with evidence of subarachnoid dissemination.

\section{Short-Term Outcome}

The complication rate was $8.3 \%$, with 1 case of postoperative hemorrhage requiring a revision, 1 superficial wound infection, and 1 postoperative urinary tract infec- 
TABLE 1. Diagnostic and intraoperative features according to tumor size and extension

\begin{tabular}{|c|c|c|c|c|c|c|}
\hline Case No. & Level & Previous Operations & Surgery & Tumor Capsule* & Subarachnoid Seeding & Radiotherapy \\
\hline 1 & L-1 & - & - & ND & No & No \\
\hline 2 & L-1 & - & GTR & Yes & No & No \\
\hline 3 & $\mathrm{~L}-2$ & 1 & GTR & No & No & No \\
\hline 4 & $\mathrm{~L}-2$ & - & GTR & Yes & No & No \\
\hline 5 & $\mathrm{~L}-2$ & - & GTR & Yes & No & No \\
\hline 6 & $\mathrm{~L}-2$ & - & GTR & Yes & No & No \\
\hline 7 & L-2 & - & GTR & Yes & No & No \\
\hline 8 & L-2 & - & GTR & Yes & No & No \\
\hline \multirow[t]{2}{*}{9} & L-2 & - & GTR & No & No & No \\
\hline & L-4, S1-2 & 1 & SR & No & Yes & Yes \\
\hline 10 & L-3 & - & GTR & Yes & No & No \\
\hline 11 & $\mathrm{~T} 11-12$ & 1 & - & ND & Yes & No \\
\hline 12 & T12-L1 & - & GTR & Yes & No & No \\
\hline 13 & T12-L1 & - & GTR & Yes & No & No \\
\hline 14 & T12-L1 & - & GTR & Yes & No & No \\
\hline 15 & T12-L1 & 1 & GTR & Yes & No & No \\
\hline 16 & L1-2 & - & GTR & Yes & No & No \\
\hline 17 & L1-2 & - & GTR & Yes & No & No \\
\hline 18 & L2-3 & - & GTR & Yes & No & No \\
\hline 19 & L2-3 & - & GTR & Yes & No & No \\
\hline 20 & L2-3 & - & GTR & Yes & No & No \\
\hline 21 & L3-4 & - & GTR & Yes & No & No \\
\hline 22 & S1-2 & 1 & - & ND & Yes & No \\
\hline 23 & S2-3 & - & GTR & No & No & No \\
\hline 24 & T11-L1 & - & GTR & Yes & No & No \\
\hline 25 & T11-L1 & 2 & - & ND & Yes & No \\
\hline 26 & T12-L2 & - & GTR & Yes & No & No \\
\hline 27 & T12-L2 & - & GTR & Yes & No & No \\
\hline 28 & T12-L2 & 1 & - & ND & No & No \\
\hline 29 & L1-3 & - & GTR & Yes & No & No \\
\hline 30 & L1-3 & - & GTR & Yes & No & No \\
\hline 31 & L2-4 & 1 & GTR & No & Yes & No \\
\hline 32 & L5-S2 & - & SR & No & No & Yes \\
\hline 33 & L5-S2 & - & GTR & Yes & No & No \\
\hline 34 & S1-3 & - & - & ND & No & No \\
\hline 35 & T-10, L4-5 & 1 & GTR & No & Yes & Yes \\
\hline 36 & T11-L3 & - & - & ND & Yes & No \\
\hline 37 & T12-L4 & - & SR & No & No & No \\
\hline 38 & L4-S3 & 2 & SR & No & Yes & Yes \\
\hline 39 & T12-S1 & - & - & ND & Yes & No \\
\hline 40 & T12-S2 & - & SR & No & No & No \\
\hline 41 & T11-S3 & 1 & SR & No & Yes & Yes \\
\hline \multirow[t]{2}{*}{42} & L2-S2 & 1 & SR & No & Yes & No \\
\hline & L2-S2 & 2 & SR & No & Yes & Yes \\
\hline
\end{tabular}

$\mathrm{ND}=$ not determined; $\mathrm{SR}=$ subtotal removal.

* Presence or absence could only be determined intraoperatively. 

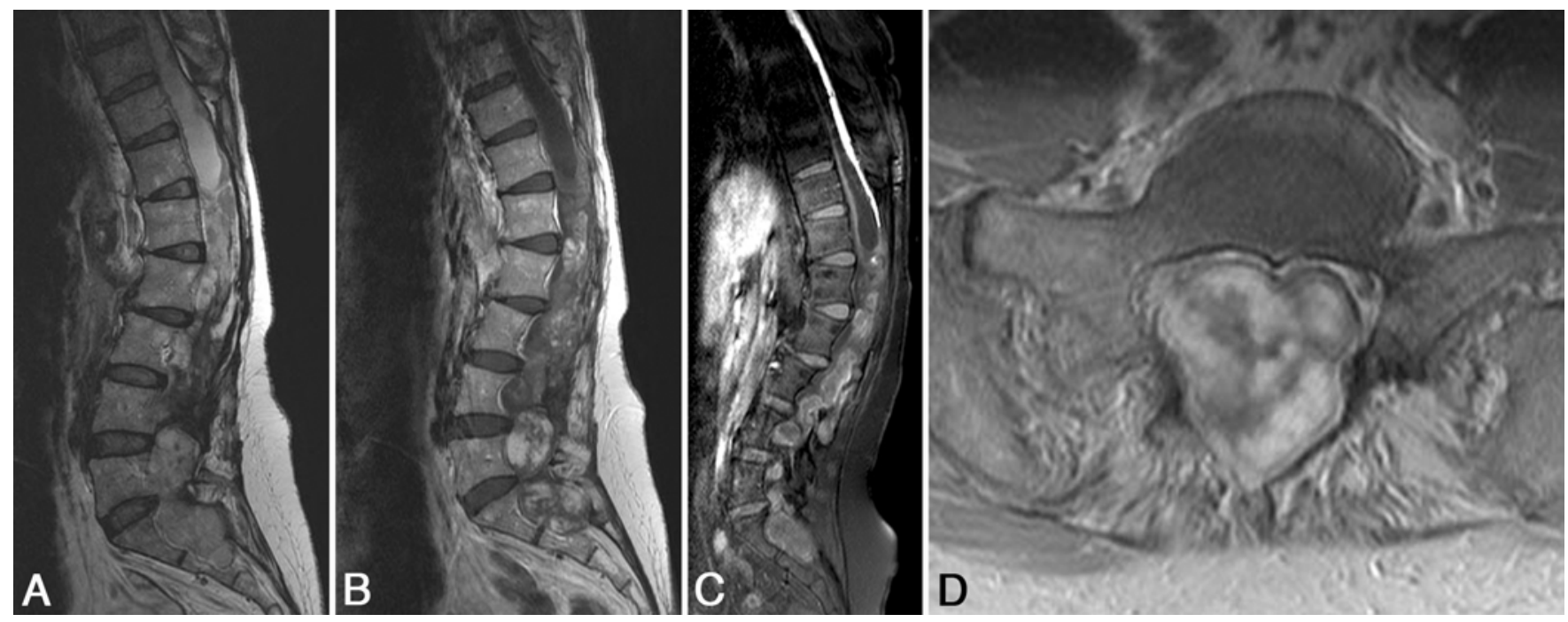

FIG. 2. Case of a 50-year-old woman with an extensive ependymoma. The tumor had been partially removed at another institution and postlaminectomy kyphosis had developed before presenting in 1986, when a second incomplete resection was undertaken followed by radiotherapy. The tumor progressed, leading to a complete paraplegia below the L- 5 level 13 years later. The patient has remained mobile with a walker and has shown no evidence of subarachnoid dissemination. A: Current sagittal T2-weighted MR image showing the tumor fills the entire sacral and lumbar canal up to the L-1 level, causing syringomyelia up into the cervical spine. B: Sagittal T1-weighted MR image with contrast showing a patchy enhancement and penetration of the tumor through the dura in the sacrum. The patient developed a chronic low pressure syndrome due to permanent loss of CSF. C: After intrathecal application of gadolinium, the T1-weighted image with fat suppression shows a subtotal block of contrast at T-12, with some contrast passing down into the sacrum. D: At L-3, the T1-weighted MR image with fat suppression indicates contrast in posterior soft tissues outside the dural sac. The patient refused another attempt of tumor resection and underwent a dural reconstruction in this area.

tion. Transient postoperative neurological deterioration was observed in 3 patients $(8.3 \%)$ and was restricted to patients with tumors without capsules, of whom 2 were undergoing secondary interventions.

Permanent surgical morbidity affected 3 patients with encapsulated tumors in the form of persistent sphincter disturbances, while 7 patients demonstrated no postoperative neurological changes and the remaining 26 operations were followed by improvements. The postoperative clinical results for the first 12 months are presented in Table 3. Patients with encapsulated ependymomas experienced a very gratifying postoperative course, with all preoperative scores returning almost to normal within the 1st postoperative year. For unencapsulated tumors, which also tended to be considerably larger, similar postoperative improvements were observed. As patients of the latter group presented with more severe preoperative neurological deficits, their scores after 1 year remained lower than those for the group with encapsulated tumors.

\section{Long-Term Outcome}

During long-term follow-up, 9 tumors recurred, of which 4 were resected completely, with all but 2 patients undergoing a secondary intervention. The overall recurrence rates were $6.6 \%, 19.0 \%$, and $37.0 \%$ after 1,10 , and 20 years, respectively.

Recurrences were restricted almost entirely to tumors without a capsule, with recurrence rates of $15.6 \%, 32.5 \%$, and $66.2 \%$ after 1,10 , and 20 years, respectively, compared with 1 recurrence after 9 years for encapsulated tumors, corresponding to rates of $9.1 \%$ after 10 and 20 years. This difference between encapsulated and unencapsulated tumor recurrences was statistically significant $(\mathrm{p}=0.011$, log-rank test) (Fig. 3). Regarding the effectiveness of postoperative radiotherapy for unencapsulated ependymomas, there was a tendency to prolong progression-free survival with radiotherapy. Whereas without radiotherapy, $40.7 \%$ of patients experienced progressive neurological deterioration within 5 years and all patients within 13 years, no

TABLE 2. Presenting symptoms*

\begin{tabular}{lcccccc}
\hline \multicolumn{1}{c}{ Group } & Hypesthesia & Dysesthesia & Pain & Motor Weakness & Gait Ataxia & Sphincter Disturbance \\
\hline Ependymoma w/ tumor capsule & 29 & 14 & 86 & 29 & 29 & 29 \\
\hline Ependymoma w/o tumor capsule & 50 & 29 & 86 & 64 & $50 \dagger$ & $86 \dagger$ \\
\hline All & 40 & 19 & 86 & 45 & 40 & 50 \\
\hline * Values are percentage of patients. & & & & & & \\
$\dagger$ Significant difference $(p<0.05)$. &
\end{tabular}


TABLE 3. Preoperative and postoperative neurological scores*

\begin{tabular}{|c|c|c|c|}
\hline Symptom & $\begin{array}{l}\text { Ependymoma } \\
\text { w/ Capsule }\end{array}$ & $\begin{array}{l}\text { Ependymoma } \\
\text { w/o Capsule }\end{array}$ & All \\
\hline \multicolumn{4}{|l|}{ Hypesthesia } \\
\hline Preop & $4.5 \pm 0.9$ & $3.6 \pm 1.3$ & $4.1 \pm 1.1$ \\
\hline Postop & $4.6 \pm 0.6$ & $3.8 \pm 1.2$ & $4.2 \pm 1.0$ \\
\hline $3 \mathrm{mos}$ & $4.7 \pm 0.6$ & $3.9 \pm 1.3$ & $4.3 \pm 1.0$ \\
\hline $6 \mathrm{mos}$ & $4.7 \pm 0.6$ & $4.1 \pm 1.1$ & $4.4 \pm 0.9$ \\
\hline $1 \mathrm{yr}$ & $4.8 \pm 0.6$ & $4.1 \pm 1.1$ & $4.4 \pm 0.9$ \\
\hline \multicolumn{4}{|l|}{ Dysesthesia } \\
\hline Preop & $4.9 \pm 0.4$ & $4.5 \pm 0.7$ & $4.7 \pm 0.5$ \\
\hline Postop & $4.9 \pm 0.4$ & $4.4 \pm 0.8$ & $4.7 \pm 0.6$ \\
\hline $3 \mathrm{mos}$ & $4.9 \pm 0.4$ & $4.5 \pm 0.7$ & $4.7 \pm 0.5$ \\
\hline $6 \mathrm{mos}$ & $4.9 \pm 0.4$ & $4.6 \pm 0.7$ & $4.8 \pm 0.5$ \\
\hline $1 \mathrm{yr}$ & $4.9 \pm 0.3$ & $4.6 \pm 0.7$ & $4.8 \pm 0.5$ \\
\hline \multicolumn{4}{|l|}{ Pain } \\
\hline Preop & $3.2 \pm 0.9$ & $3.4 \pm 1.0$ & $3.3 \pm 0.9$ \\
\hline Postop & $3.9 \pm 0.5$ & $4.0 \pm 0.7$ & $3.9 \pm 0.6$ \\
\hline $3 \mathrm{mos}$ & $4.3 \pm 0.7$ & $4.2 \pm 0.6$ & $4.3 \pm 0.7$ \\
\hline $6 \mathrm{mos}$ & $4.3 \pm 0.7$ & $4.1 \pm 0.7$ & $4.2 \pm 0.7$ \\
\hline $1 \mathrm{yr}$ & $4.4 \pm 0.7$ & $3.9 \pm 0.9$ & $4.2 \pm 0.8$ \\
\hline \multicolumn{4}{|l|}{ Motor weakness } \\
\hline Preop & $4.7 \pm 0.6$ & $3.6 \pm 1.0$ & $4.2 \pm 0.9$ \\
\hline Postop & $4.7 \pm 0.6$ & $3.5 \pm 1.3$ & $4.2 \pm 1.1$ \\
\hline $3 \mathrm{mos}$ & $4.9 \pm 0.4$ & $3.7 \pm 1.3$ & $4.4 \pm 1.0$ \\
\hline $6 \mathrm{mos}$ & $4.9 \pm 0.3$ & $3.8 \pm 1.2$ & $4.5 \pm 1.0$ \\
\hline $1 \mathrm{yr}$ & $4.9 \pm 0.3$ & $3.9 \pm 1.0$ & $4.5 \pm 0.9$ \\
\hline \multicolumn{4}{|l|}{ Gait ataxia } \\
\hline Preop & $4.8 \pm 0.4$ & $3.6 \pm 1.1$ & $4.3 \pm 1.0$ \\
\hline Postop & $4.7 \pm 0.7$ & $3.5 \pm 1.4$ & $4.2 \pm 1.2$ \\
\hline $3 \mathrm{mos}$ & $4.7 \pm 0.6$ & $3.8 \pm 1.3$ & $4.4 \pm 1.1$ \\
\hline $6 \mathrm{mos}$ & $4.9 \pm 0.4$ & $3.9 \pm 1.3$ & $4.5 \pm 1.0$ \\
\hline $1 \mathrm{yr}$ & $4.9 \pm 0.4$ & $4.0 \pm 1.1$ & $4.5 \pm 0.8$ \\
\hline \multicolumn{4}{|c|}{ Bladder dysfunction } \\
\hline Preop & $4.6 \pm 0.7$ & $3.0 \pm 1.3$ & $4.0 \pm 1.3$ \\
\hline Postop & $4.6 \pm 0.9$ & $2.7 \pm 1.3$ & $3.8 \pm 1.4$ \\
\hline $3 \mathrm{mos}$ & $4.6 \pm 0.9$ & $3.1 \pm 1.3$ & $4.0 \pm 1.3$ \\
\hline $6 \mathrm{mos}$ & $4.6 \pm 0.9$ & $3.2 \pm 1.3$ & $4.0 \pm 1.3$ \\
\hline $1 \mathrm{yr}$ & $4.6 \pm 0.9$ & $3.3 \pm 1.4$ & $4.1 \pm 1.3$ \\
\hline
\end{tabular}

* Values are mean \pm standard deviation.

such deterioration was observed before 12 years after radiotherapy (Fig. 4). The low number of patients, however, prevented this difference from reaching a significant level.

Multiple regression analysis was used to determine independent factors predicting long progression-free survival $(\mathrm{r}=0.5838, \mathrm{p}=0.024)$. Encapsulated tumors $(0.5407)$ and a GTR $(0.4759)$ were the strongest factors in this respect, followed by postoperative radiotherapy $(0.2331)$ and short preoperative history (0.1955). Patient age, sex, tumor size, or severity of preoperative symptoms had no influence on progression-free survival.

Five patients died during follow-up: 3 deaths were unrelated to the ependymoma operated on (supratentorial glioblastoma, metastasized bladder carcinoma, and cardiac failure), while 2 deaths involved unencapsulated tumors and occurred at 216 and 287 months after surgery from urosepsis and widespread tumor dissemination, re-

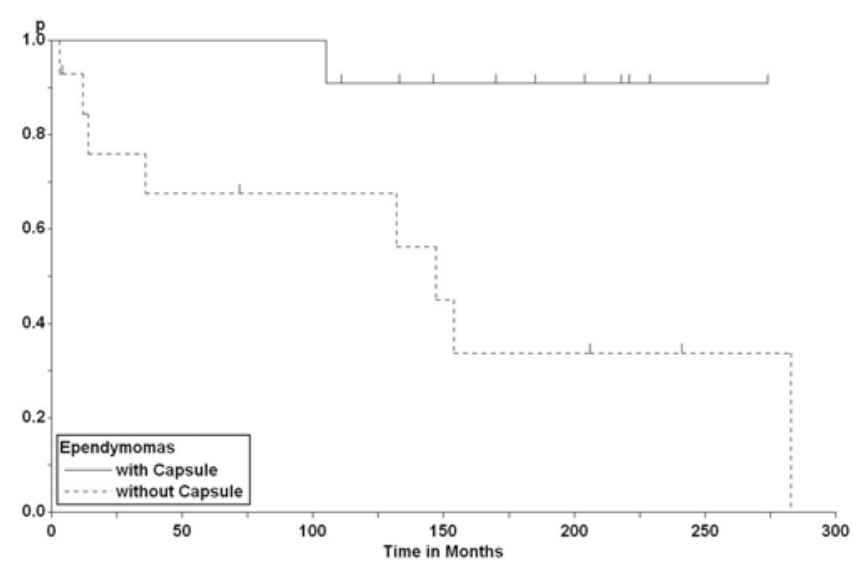

FIG. 3. Kaplan-Meier analysis of tumor recurrence rates for encapsulated and unencapsulated ependymomas ( $p=0.011$, log-rank test).

spectively. This corresponds to a 20-year survival rate of $88.9 \%$ according to Kaplan-Meier analysis.

\section{Discussion \\ Clinical Presentation and Diagnosis}

Myxopapillary ependymomas of the filum terminale are rare spinal tumors. The study presented here extended over 32 years to collect a series of 42 patients representing $4.5 \%$ of all intradural tumors in that period. In a series of 1013 patients with intra- and extramedullary spinal cord tumors, Epstein's group identified a similar rate of 5.1\% for myxopapillary ependymomas. ${ }^{6}$ With the exception of 3 children, all patients were adults. Males outnumbered females at a ratio of 1.5:1. The most common presenting symptom was back pain, ${ }^{6,13,23,35}$ mostly of dull intensity and restricted to the lower back. Some patients reported a pseudoradicular component as well. Neurological deficits were of mild to moderate degree in the majority of patients. Only $7 \%$ of patients were unable to walk, and $9 \%$ required urinary catheterization at the time of presentation (Table 2). This is in striking contrast to the often considerable size of these tumors and is an indicator of their usually slow growth. On average, it took about 3 years before patients presented and the diagnosis was made. Compared with the most common extramedullary tumors-neurinomas ( $\mathrm{n}=170 ; 21 \pm 31$ months $)$ and meningiomas $(\mathrm{n}=189$; $17 \pm 26$ months) - this time period was significantly longer $(p=0.01)$. More aggressive clinical presentations have been reported in pediatric series. ${ }^{6}$ Acute presentations with a sudden onset of severe pain are rare and related to tumor hemorrhages..$^{15,37}$ This was encountered in only 1 patient in this series.

As expected, the preoperative clinical history tended to be longer for larger tumors. For those patients choosing to decline surgery, the clinical history tended to be considerably longer, reaching almost 10 years on average. This appeared to be the major reason to refuse an operation.

Interestingly, almost all ependymomas extending over a maximum of 3 spinal segments displayed a capsule, whereas the majority of those extending over 4 or more segments did not. This finding may suggest that ependy- 


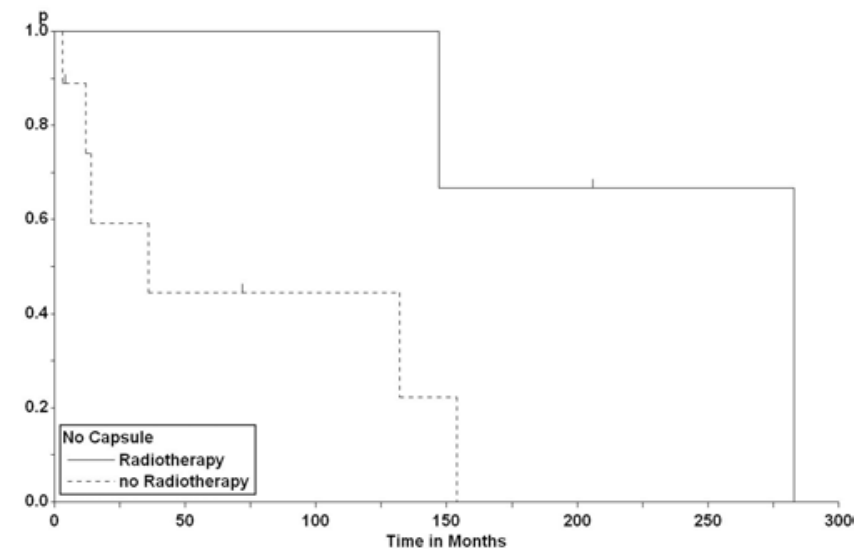

FIG. 4. Kaplan-Meier analysis demonstrating progression-free survival rates for patients with unencapsulated tumors with and without radiotherapy (not significant, log-rank test).

momas display a tumor capsule at the beginning, which is often quite fragile. Once they reach a certain size, further growth and tumor hemorrhages may lead to rupture of the capsule, infiltration of surrounding nerve roots, and even dissemination into the subarachnoid space. With the exception of 2 unoperated patients presenting with large ependymomas, all patients demonstrating subarachnoid dissemination had undergone incomplete tumor removal, suggesting that dissemination is an iatrogenic phenomenon in the majority of instances. ${ }^{28}$ Dissemination is unusual in previously unoperated patients ${ }^{16,18,24,36}$ and was restricted to large tumors without capsules in this series. Therefore, the entire spinal axis should be examined on MRI whenever a large tumor extending over more than 4 spinal segments or a recurrent tumor is encountered.

The preoperative diagnosis requires MR images with and without contrast. Unlike intramedullary ependymomas, which may display various appearances on MRI, ${ }^{27}$ ependymomas of the filum terminale demonstrate a more uniform appearance, are isodense to cord tissue on T1weighted images, and are hyperintense on T2-weighted images with strong contrast enhancement ${ }^{40}$ (Fig. 1). Heterogeneous contrast enhancement has been reported to indicate unencapsulated tumors ${ }^{34}$ (Fig. 2).

With large tumors filling the entire lumbar canal, the diagnosis of a myxopapillary ependymoma is straightforward. Especially in the sacral region, large tumors may penetrate the dura and lead to local bone destruction ${ }^{32}$ (Fig. 2). Smaller ependymomas, however, are impossible to distinguish from other extramedullary tumors such as schwannomas, for instance. ${ }^{21}$

\section{Surgical Management}

It is the general policy of the 2 institutions involved in the series to recommend surgery for intradural spinal tumors as soon as the diagnosis is made and symptoms are present. ${ }^{21}$ Myxopapillary ependymomas should be treated according to this policy as well, aiming for a GTR regardless of their size. ${ }^{12,23}$ The strategy for small tumors is to attempt in toto removal, with resection of the filum. With large tumors filling out the spinal canal completely and/ or extending cranially beyond the conus, en bloc removals are impossible without significant neurological deficits and should not be attempted. In such instances, the tumor mass has to be debulked first. Debulking, however, raises the issue of subarachnoid spreading of tumor particles. Therefore, this must be done with great care. Placing cottonoids around the tumor appears to minimize this risk and also helps to protect nerve roots once they have been dissected away from the tumor. An assistant operating a second suction can be very helpful to avoid tumor dissemination during resection. Whenever a tumor displays a capsule, nerve roots will be displaced by the tumor mass rather than be encased in it. Once the mass has been reduced, the filum can be transected distally and then lifted up with the tumor remnant to continue dissection on the ventral side. To identify the exact position of the conus, the cranial pole of the tumor should then be dissected off the spinal cord surface and followed distally. By exposing and dissecting the ventral tumor portion from either end, the conus position can be identified safely and the filum finally cut immediately below it (Fig. 1).

With large tumors encasing nerve roots and causing atrophy of the inner dural layer or even both layers due to the effect of extradural extensions, dissection and removal are more difficult and time consuming and dura closure and reconstruction may also become challenging (Fig. 2). Fortunately, modern imaging techniques and MRI, in particular, seem to have contributed to the lower number of patients presenting with huge tumors in recent years. In this series, the last patient to present with a huge, previously unoperated tumor was in 1999. If tumors filling out the lumbar and sacral canal are encountered, they should be referred to neurosurgeons experienced in their management. Performing biopsies or attempting just a partial resection cannot be recommended as these procedures considerably raise the risk for subarachnoid dissemination. A realistic possibility for GTR in such cases is restricted to the first surgical attempt.

Despite the difficulty, complete resection should be attempted when dissecting a de novo ependymoma encasing nerve roots. Depending on the localization and number of nerve roots appearing to be infiltrated by tumor or carrying significant tumor vessels, the surgeon may have to decide whether to resect them with the tumor, increasing the radicality of the surgery, or to preserve them, risking a local recurrence. Intraoperative monitoring and stimulation may aid in such decisions and are highly recommended.

Once the tumor is resected, dural closure can be performed with a running suture whenever the consistency of the dura allows the surgeon to do so. With atrophy of the inner dural layer, it may be advisable to add a second layer with a fascia lata graft supporting the atrophied dura. Whenever parts of the dura require reconstruction, artificial rather than autologous materials should be employed. Autologous materials tend to cause profound adhesions between nerve roots, conus, and duraplasty and should be avoided. If the dural suture appears not to be reliable, a fascia graft may then be added, as a sandwich, on top of the artificial duraplasty. In such instances, a lumbar drain should be placed as well to lower the subarachnoid pressure for at least a week to prevent a CSF fistula. 
With the use of this strategy in our patients, $77.7 \%$ of all tumors were completely resected. Incomplete resections were restricted to tumors without capsules $(61.5 \%)$, whereas all encapsulated tumors underwent GTR. Similar results were reported by Xie et al., ${ }^{39}$ who were able to resect $87 \%$ of 38 tumors completely, and Kucia et al., ${ }^{23}$ who achieved $80 \%$ complete resections in 34 patients. In other reviews, considerably lower rates for GTRs were published. Oh et al. ${ }^{30}$ reported a rate of only $58.9 \%$ for 56 operations; Akyurek et al., ${ }^{2} 60 \%$ for 35 patients; Nakamura et al., ${ }^{28} 64 \%$ for 25 operations; and Celli et al., ${ }^{9} 71 \%$ for 28 patients. In their literature review, Figueiredo et al. ${ }^{13}$ calculated a rate of $59.79 \%$ GTRs for 199 patients. None of these studies provided separate figures for encapsulated and unencapsulated ependymomas.

\section{Radiotherapy}

Radiotherapy for spinal ependymomas is a controversial issue. It is not recommended for intramedullary ependymomas. ${ }^{25}$ These tumors are best treated surgically, can be resected completely, display no subarachnoid dissemination, and carry a very low risk of recurrence. ${ }^{19}$ For extramedullary ependymomas, however, issues such as infiltration of nerve roots, which need to be preserved, or potential for subarachnoid dissemination raise the question of postoperative radiotherapy even after GTR. In this decision process, results for radiotherapy of intracranial ependymomas are of no help as ependymomas behave differently according to their site of origin. ${ }^{33}$

There appears to be no general consensus in the literature as to when radiotherapy should be applied or in what form. In a large literature review, Feldman et al., ${ }^{12}$ as well as Bagley et al. ${ }^{6}$ in their series of 52 patients or Celli et al. ${ }^{9}$ studying 28 patients, were unable to establish a benefit for postoperative radiotherapy of myxopapillary ependymomas. On the other hand, Pica et al. ${ }^{31}$ found postoperative radiotherapy to be the only independent factor in a multivariate analysis predicting a long progression-free survival in a series of 85 patients. Likewise, Nakamura et al. ${ }^{28}$ and Akyurek et al. ${ }^{2}$ recommended local radiotherapy as well as radiation for the entire spinal axis to prevent local recurrences as well as postoperative tumor dissemination, whereas Gilhuis et al. ${ }^{14}$ reserved radiotherapy for patients after incomplete resections. Some studies suggest that radiotherapy may have a particular role in pediatric patients..$^{1,4}$ In their literature review, Lonjon et al. ${ }^{26}$ calculated a recurrence rate of $33 \%$ with radiotherapy and $55 \%$ without, suggesting that radiotherapy may have a role as adjuvant therapy. In another recent literature review, radiotherapy was recommended even after GTRs, as recurrence rates were reported to be lower than rates with surgery alone. $^{13}$

In this series, only 1 recurrence was encountered after GTR of encapsulated tumors. Surgery alone appears to be curative for this subgroup, similarly to their intramedullary counterparts (Fig. 3). For unencapsulated tumors, radiotherapy was employed in 5 patients. Four of these tumors had demonstrated subarachnoid dissemination prior to surgery. The fifth patient had undergone an incomplete resection of a large ependymoma early in the series. In the long term, all patients with unencapsulated ependymomas experienced a recurrence irrespective of the amount of resection or administration of radiotherapy, according to Kaplan-Meier analysis. However, there was a trend for longer recurrence-free survival for patients after radiotherapy in this subgroup: none of these patients experienced a neurological deterioration before 12 years, whereas without radiotherapy, $41.7 \%$ of patients had shown a clinical relapse within 5 years (Fig. 4). In a multiple regression analysis, postoperative radiotherapy was an independent predictor for a long progression-free interval. With the limitation of the small number of patients involved, this study suggests that radiotherapy may have a role in patients with subarachnoid dissemination $^{5}$ and after incomplete resections of large unencapsulated tumors..$^{10,11,13,23,29,35}$ However, attempting a GTR before these tumors have reached a size spreading over more than 3 spinal segments offers the best chance for a long progression-free survival.

\section{Outcome}

The immediate postoperative outcome was very favorable for the great majority of patients of this series. ${ }^{39}$ Among 36 operations, 26 were followed by postoperative improvements. Only 7 patients experienced an unchanged status, and 3 patients underwent a permanent deterioration of bladder function after surgery. As one would expect, the results after 1 year somewhat depended on the degree of preoperative deficits and were better for encapsulated tumors, even though both subgroups benefitted from surgery, as shown in Table 3. The rate of $8.3 \%$ for permanent surgical morbidity is considerably lower than rates reported for other studies. ${ }^{34,38}$

In the long term, tumor recurrence rates are low after GTRs for encapsulated tumors ${ }^{23}$ (Fig. 3). On the other hand, for unencapsulated tumors a GTR does not prevent a recurrence. ${ }^{2}$ In their literature reviews, Feldman et al. ${ }^{12}$ and Benesch et al. ${ }^{7}$ found significantly higher recurrence rates for pediatric than for adult patients. Agbahiwe et al. ${ }^{1}$ reported recurrence rates for children after GTRs of $38.5 \%$ and $70 \%$ within 5 and 10 years, respectively, and Bagley et al. ${ }^{6}$ reported a rate of $64 \%$. In this study, only 3 pediatric patients were included. One of them experienced a recurrence related to tumor dissemination after undergoing a second attempt of GTR.

In this series, 9 tumors recurred. The overall recurrence rates according to Kaplan-Meier statistics were $6.6 \%$, $19.0 \%$, and $37.0 \%$ after 1,10 , and 20 years, respectively. Lonjon et al. ${ }^{26}$ reported a recurrence rate of $15 \%$ after complete resections according to a literature review, but without distinguishing tumors with and without capsules. In a more recent review, Figueiredo et al. ${ }^{13}$ reported higher overall recurrence rates of $21 \%$ and $34 \%$ after 5 and 10 years, respectively. Most studies, however, do not use statistical techniques such as Kaplan-Meier analysis to report recurrence rates and only provide the numbers of recurrent tumors: Xie et al. ${ }^{39}$ observed 6 recurrences among 38 patients; Kucia et al., ${ }^{23} 3$ recurrences in 34 patients; Al-Habib et al., 3 recurrences among 18 patients; and Akyurek et al., ${ }^{2} 5$ recurrences among 11 patients. Compared with intramedullary ependymomas, recurrence rates after GTRs are higher for ependymomas of the filum terminale..$^{8,19,30}$

In this series, recurrences were found almost exclusive- 
ly after surgery on unencapsulated tumors, with all tumors having recurred within 24 years after surgery (Fig. 3). Likewise, Xie et al. ${ }^{39}$ found unencapsulated tumors, those extending over more than 2 spinal levels, and those invading the sacral canal to bear a worse long-term prognosis. Celli et al. ${ }^{9}$ considered a long preoperative clinical history and adherence to conus and nerve roots as unfavorable features associated with higher recurrence rates-characteristics more common in unencapsulated tumors. Whether a GTR of an unencapsulated tumor may prevent a recurrence in the long term cannot be evaluated in this series, as the maximum follow-up for this subset of patients was limited to 6 years.

Five patients died during follow-up in this series. Two tumor-related deaths occurred in the group of patients with unencapsulated tumors: death was due to widespread dissemination in 1 case (at 287 months after surgery) and urosepsis in the other (at 216 months after surgery). The corresponding 20 -year survival rate was $88.9 \%$. No comparable data have been published in the literature. The fact, however, that a benign tumor of WHO Grade I may be responsible for a patient's death unless it is diagnosed early and completely resected indicates that this pathology should be addressed surgically irrespective of the sometimes indolent course for long periods of time.

\section{Conclusions}

In this series, myxopapillary ependymomas were operated on with low surgical morbidity and favorable neurological results. Despite their often considerable size, these tumors should be resected completely as soon as they cause symptoms. GTR of encapsulated ependymomas appears to be curative according to this series. With increasing size of these tumors, violations of the tumor capsule, infiltration of nerve roots, and subarachnoid dissemination limit the resectability of these tumors and the chances for long-term progression-free survival. The role of postoperative radiotherapy warrants further investigations. According to recent reviews and this report, radiotherapy may be considered for patients after incomplete resections of unencapsulated tumors and/or given evidence of subarachnoid dissemination.

\section{Acknowledgments}

The author wishes to express his gratitude to Prof. Madjid Samii, who supervised the author's neurosurgical training, taught the author how to operate on these challenging lesions, and enabled this study as well as the establishment of the spinal cord database by attracting large numbers of patients with spinal cord pathologies to his neurosurgical clinic in Hannover. The author wishes to acknowledge the leadership of Drs. Madjid Samii (1977-2002) and Michael Gaab (2003-2013) during their respective tenures as Chairman of the Neurosurgical Department in Hannover (Nordstadt Hospital).

\section{References}

1. Agbahiwe HC, Wharam M, Batra S, Cohen K, Terezakis SA: Management of pediatric myxopapillary ependymoma: the role of adjuvant radiation. Int J Radiat Oncol Biol Phys 85:421-427, 2013
2. Akyurek S, Chang EL, Yu TK, Little D, Allen PK, McCutcheon I, et al: Spinal myxopapillary ependymoma outcomes in patients treated with surgery and radiotherapy at M.D. Anderson Cancer Center. J Neurooncol 80:177-183, 2006

3. Al-Habib A, Al-Radi OO, Shannon P, Al-Ahmadi H, Petrenko Y, Fehlings MG: Myxopapillary ependymoma: correlation of clinical and imaging features with surgical resectability in a series with long-term follow-up. Spinal Cord 49:10731078, 2011

4. Al-Halabi H, Montes JL, Atkinson J, Farmer JP, Freeman CR: Adjuvant radiotherapy in the treatment of pediatric my xopapillary ependymomas. Pediatr Blood Cancer 55:639-643, 2010

5. Andoh H, Kawaguchi Y, Seki S, Asanuma Y, Fukuoka J, Ishizawa S, et al: Multi-focal myxopapillary ependymoma in the lumbar and sacral regions requiring cranio-spinal radiation therapy: a case report. Asian Spine J 5:68-72, 2011

6. Bagley CA, Wilson S, Kothbauer KF, Bookland MJ, Epstein F, Jallo GI: Long term outcomes following surgical resection of myxopapillary ependymomas. Neurosurg Rev 32:321334, 2009

7. Benesch M, Frappaz D, Massimino M: Spinal cord ependymomas in children and adolescents. Childs Nerv Syst 28:2017-2028, 2012

8. Boström A, von Lehe M, Hartmann W, Pietsch T, Feuss M, Boström JP, et al: Surgery for spinal cord ependymomas: outcome and prognostic factors. Neurosurgery 68:302-309, 2011

9. Celli P, Cervoni L, Cantore G: Ependymoma of the filum terminale: treatment and prognostic factors in a series of 28 cases. Acta Neurochir (Wien) 124:99-103, 1993

10. Chao ST, Kobayashi T, Benzel E, Reddy CA, Stevens GH, Prayson RA, et al: The role of adjuvant radiation therapy in the treatment of spinal myxopapillary ependymomas. J Neurosurg Spine 14:59-64, 2011

11. de Jong L, Calenbergh FV, Menten J, van Loon J, De Vleeschouwer S, Plets C, et al: Ependymomas of the filum terminale: The role of surgery and radiotherapy. Surg Neurol Int 3:76, 2012

12. Feldman WB, Clark AJ, Safaee M, Ames CP, Parsa AT: Tumor control after surgery for spinal myxopapillary ependymomas: distinct outcomes in adults versus children: a systematic review. J Neurosurg Spine 19:471-476, 2013

13. Figueiredo N, Brooks N, Resnick DK: Evidence-based review and guidelines for the management of myxopapillary and intramedullary ependymoma. J Neurosurg Sci 57:327-341, 2013

14. Gilhuis HJ, Kappelle AC, Beute G, Wesseling P, Grotenhuis A, Boerman RH: Radiotherapy for partially resected spinal ependymomas: a retrospective study of 60 cases. Oncol Rep 10:2079-2082, 2003

15. Heuer GG, Stiefel MF, Bailey RL, Schuster JM: Acute paraparesis from hemorrhagic spinal ependymoma: diagnostic dilemma and surgical management. Report of two cases and review of the literature. J Neurosurg Spine 7:652-655, 2007

16. Iunes EA, Stávale JN, de Cássia Caldas Pessoa R, Ansai R, Onishi FJ, de Paiva Neto MA, et al: Multifocal intradural extramedullary ependymoma. Case report. J Neurosurg Spine 14:65-70, 2011

17. Kaplan EL, Meier P: Nonparametric estimation from incomplete observations. J Am Stat Assoc 53:457-481, 1958

18. Khalatbari MR, Jalaeikhoo H, Hamidi M, Moharamzad Y: Craniospinal dissemination of filum myxopapillary ependymoma following spinal trauma: case report and literature review. Childs Nerv Syst 29:149-152, 2013

19. Klekamp J: Treatment of intramedullary tumors: analysis of surgical morbidity and long-term results. J Neurosurg Spine 19:12-26, 2013

20. Klekamp J, Samii M: Introduction of a score system for the 
clinical evaluation of patients with spinal processes. Acta Neurochir (Wien) 123:221-223, 1993

21. Klekamp J, Samii M: Surgery of Spinal Tumors. Berlin: Springer, 2007

22. Klekamp J, Samii M: Syringomyelia - Diagnosis and Treatment. Berlin: Springer, 2001

23. Kucia EJ, Maughan PH, Kakarla UK, Bambakidis NC, Spetzler RF: Surgical technique and outcomes in the treatment of spinal cord ependymomas: part II: myxopapillary ependymoma. Neurosurgery 68 (1 Suppl Operative):90-94, 2011

24. Landriel F, Ajler P, Tedesco N, Bendersky D, Vecchi E: Multicentric extramedullary myxopapillary ependymomas: Two case reports and literature review. Surg Neurol Int 3:102, 2012

25. Lee SH, Chung CK, Kim CH, Yoon SH, Hyun SJ, Kim KJ, et al: Long-term outcomes of surgical resection with or without adjuvant radiation therapy for treatment of spinal ependymoma: a retrospective multicenter study by the Korea Spinal Oncology Research Group. Neuro Oncol 15:921-929, 2013

26. Lonjon M, Von Langsdorf D, Lefloch S, Rahbi M, Rasendrarijao D, Michiels JF, et al: [Factors influencing recurrence and role of radiotherapy in filum terminale ependymomas. 14 cases and review of the literature.] Neurochirurgie 47:423429, 2001 (Fr)

27. Miyazawa N, Hida K, Iwasaki Y, Koyanagi I, Abe H: MRI at $1.5 \mathrm{~T}$ of intramedullary ependymoma and classification of pattern of contrast enhancement. Neuroradiology 42:828 832,2000

28. Nakamura M, Ishii K, Watanabe K, Tsuji T, Matsumoto M, Toyama Y, et al: Long-term surgical outcomes for myxopapillary ependymomas of the cauda equina. Spine (Phila Pa 1976) 34:E756-E760, 2009

29. Oh MC, Ivan ME, Sun MZ, Kaur G, Safaee M, Kim JM, et al: Adjuvant radiotherapy delays recurrence following subtotal resection of spinal cord ependymomas. Neuro Oncol 15:208-215, 2013

30. Oh MC, Tarapore PE, Kim JM, Sun MZ, Safaee M, Kaur G, et al: Spinal ependymomas: benefits of extent of resection for different histological grades. J Clin Neurosci 20:1390-1397, 2013

31. Pica A, Miller R, Villà S, Kadish SP, Anacak Y, Abusaris H, et al: The results of surgery, with or without radiotherapy, for primary spinal myxopapillary ependymoma: a retrospective study from the rare cancer network. Int J Radiat Oncol Biol Phys 74:1114-1120, 2009
32. Quraishi NA, Wolinsky JP, Bydon A, Witham T, Gokaslan ZL: Giant destructive myxopapillary ependymomas of the sacrum. J Neurosurg Spine 12:154-159, 2010

33. Raghunathan A, Wani K, Armstrong TS, Vera-Bolanos E, Fouladi M, Gilbertson R, et al: Histological predictors of outcome in ependymoma are dependent on anatomic site within the central nervous system. Brain Pathol 23:584-594, 2013

34. Sakai Y, Matsuyama Y, Katayama Y, Imagama S, Ito Z, Wakao N, et al: Spinal myxopapillary ependymoma: neurological deterioration in patients treated with surgery. Spine (Phila Pa 1976) 34:1619-1624, 2009

35. Schweitzer JS, Batzdorf U: Ependymoma of the cauda equina region: diagnosis, treatment, and outcome in 15 patients. Neurosurgery 30:202-207, 1992

36. Straus D, Tan LA, Takagi I, O'Toole JE: Disseminated spinal myxopapillary ependymoma in an adult at initial presentation: a case report and review of the literature. Br J Neurosurg 28:691-693, 2014

37. Tait MJ, Chelvarajah R, Garvan N, Bavetta S: Spontaneous hemorrhage of a spinal ependymoma: a rare cause of acute cauda equina syndrome: a case report. Spine (Phila Pa 1976) 29:E502-E505, 2004

38. Wostrack M, Shiban E, Obermueller T, Gempt J, Meyer B, Ringel F: Conus medullaris and cauda equina tumors: clinical presentation, prognosis, and outcome after surgical treatment: clinical article. J Neurosurg Spine 20:335-343, 2014

39. Xie TH, Chen XZ, Qian J, Lu YC, Jiang YK, Zhang L, et al: Surgery for primary filum terminale ependymomas: outcome and prognostic factors. CNS Neurosci Ther 20:131-139, 2014

40. Yuh EL, Barkovich AJ, Gupta N: Imaging of ependymomas: MRI and CT. Childs Nerv Syst 25:1203-1213, 2009

\section{Supplemental Information \\ Companion Paper}

Klekamp J: Spinal ependymomas. Part 1: Intramedullary ependymomas. DOI: 10.3171/2015.5.FOCUS 15161.

\section{Correspondence}

Jörg Klekamp, Department of Neurosurgery, Christliches Krankenhaus, Danziger Str. 2, Quakenbrück 49610, Germany. email: j.klekamp@ckq-gmbh.de. 